\title{
Dimensions of affective semantic meaning — behavioral and evoked potential correlates in Chinese subjects
}

\author{
W. Skrandies ${ }^{\mathrm{a}, *}$, M.J. Chiu ${ }^{\mathrm{b}}$ \\ anstitute of Physiology, Justus-Liebig University, Aulweg 129, 35392 Giessen, Germany \\ ${ }^{\mathrm{b}}$ Department of Neurology, National Taiwan University Hospital, College of Medicine, National Taiwan University, Taipei, Taiwan
}

Received 6 December 2002; received in revised form 21 January 2003; accepted 27 January 2003

\begin{abstract}
The affective meaning of words can be quantified statistically by the 'semantic differential technique'. We studied a total of 55 Chinese adults in two experiments: first, 210 nouns were rated by 32 subjects. Factor analysis on these data yielded three independent semantic dimensions. Semantically unique words were used in electrophysiological experiments in another group of 23 healthy right-handed adults. Words of similar physical appearance belonging to different semantic classes were presented visually in random order. The electrocardiogram was recorded in 32 channels. Evoked activity was computed for each semantic class. Significant differences in electrical brain activation between semantic word classes were seen as early as $80 \mathrm{~ms}$ after stimulus onset confirming related findings in German subjects. These results illustrate similar early neural activation in subject groups of different language and culture.

(C) 2003 Elsevier Science Ireland Ltd. All rights reserved.
\end{abstract}

Keywords: Evoked Potential; Brain Electrical Topography; Language; Semantic Meaning; Semantic Differential

It has been shown that the recording of electrical brain activity with high time resolution elicited by linguistic material yields sensitive neuronal indicators of human language processing $[11,18]$. In an earlier report from our laboratory we demonstrated that semantic meaning of words influences already very early stages of processing as reflected by changes of evoked potential fields [14]. This is in contrast to studies concerned with recordings of brain activity during language processing that centered on the socalled N400 component which occurs at long latency after the presentation of a word which unexpectedly closes a sentence [7], and relates to the comprehension of sentences or jokes [1,3].

According to the pioneering work of Osgood et al. [10], also in Hongkong Chinese the affective meaning of words can be quantified by the 'semantic differential technique' that results in statistically defined independent dimensions where every word is uniquely located on the three dimensions evaluation (E, good-bad), potency (P,

\footnotetext{
* Corresponding author. Tel.: +49-641-99-47270; fax: +49-641-9947279 .

E-mail address: wolfgang.skrandies@physiologie.med.uni-giessen.de (W. Skrandies).
}

strong-weak), and activity (A, active-passive). Brain potentials elicited during reading of single German words show systematic influences of meaning, and these effects occur as early as $100 \mathrm{~ms}$ [14]. In the present study we extend such experiments to Chinese adults. We are concerned with the question of whether the semantic dimensions are similar in German and Chinese subjects, and whether early electrophysiological effects can also be observed with different language material in subjects with a vastly different cultural background.

A total of 55 Chinese adults participated in two experiments: first, 210 nouns were rated on 12 adjective scales of opposite descriptors by 32 subjects (mean age 29.3; SD 9.6 years; 27 females, five males). All words consisted of two characters and had a comparable frequency of occurrence in the Chinese language [6]. Principal component analysis on the questionnaires extracted three varimax-rotated components which corresponded to the $\mathrm{E}$, $\mathrm{P}$, and A dimensions of Osgood et al. [9,10]. Component scores of each word were computed, and words that scored highly positive (or negative) on one component $(>0.70)$ but had only low scores on the other two dimensions were selected (i.e., stimuli were clearly associated with only one 
of the semantic dimensions). This resulted in six semantic word classes which were used subsequently.

For electrophysiological experiments, list were prepared that contained a total of 1440 words stemming from the six classes in randomized order. Twenty-three different healthy volunteers (mean age 29.8; SD 6.0 years; 13 females, ten males) with Chinese as native language were studied. All subjects were right-handed [5]. The electrocardiogram (EEG) was recorded in 32 channels simultaneously from an array of electrodes located at the following positions of the International 10/20 System: $\mathrm{Fp}_{1}, \mathrm{Fp}_{2}, \mathrm{Fp}_{\mathrm{z}}, \mathrm{F}_{4}, \mathrm{C}_{4}, \mathrm{P}_{4}, \mathrm{O}_{2}$, $\mathrm{F}_{8}, \mathrm{~T}_{8}, \mathrm{TP}_{8}, \mathrm{~F}_{3}, \mathrm{C}_{3}, \mathrm{P}_{3}, \mathrm{O}_{1}, \mathrm{~F}_{7}, \mathrm{~T}_{7}, \mathrm{TP}_{7}, \mathrm{~F}_{\mathrm{z}}, \mathrm{C}_{\mathrm{z}}, \mathrm{CP}_{\mathrm{z}}, \mathrm{P}_{\mathrm{z}}, \mathrm{PO}_{\mathrm{z}}$, $\mathrm{FC}_{2}, \mathrm{C}_{2}, \mathrm{CP}_{2}, \mathrm{FC}_{6}, \mathrm{CP}_{6}, \mathrm{FC}_{1}, \mathrm{C}_{1}, \mathrm{CP}_{1}, \mathrm{FC}_{5}, \mathrm{CP}_{5}$ (Fig. 1). Linked ears served as recording reference; for analysis all signals were referred to the computed average reference. Data acquisition was preformed with a NK Neurofax EEG 1000 system (bandpass: $0.1-60 \mathrm{~Hz}$; sampling rate: $200 \mathrm{~Hz}$ ). For computing event-related potentials, the class of each stimulus presented was coded by a trigger signal. Stimuli were presented according to the randomized lists described above sequentially on a computer monitor for $1 \mathrm{~s}$ each. Viewing distance was $1 \mathrm{~m}$, all stimuli were of high contrast ( $>95 \%, 43 \mathrm{~lx}$ mean luminosity) and subtended $1.43 \times 2.86^{\circ}$. Size was similar for all word classes. The subjects' task was to visualize the words, and recall was tested at the end of the experiment by a questionnaire. The performance on this task was routinely checked in order to

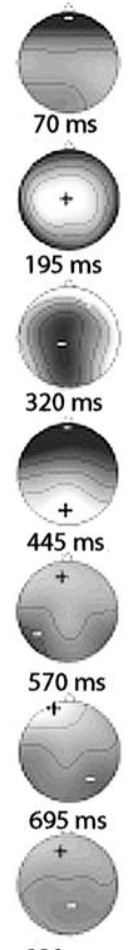

$820 \mathrm{~ms}$
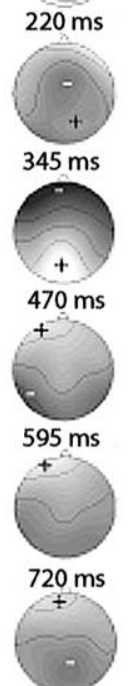

$845 \mathrm{~ms}$

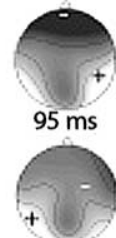

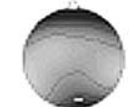

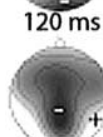

$245 \mathrm{~ms}$
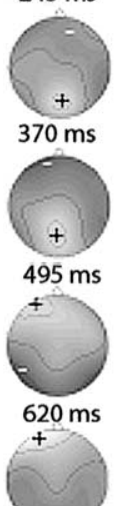

$745 \mathrm{~ms}$

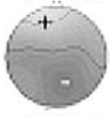

$870 \mathrm{~ms}$
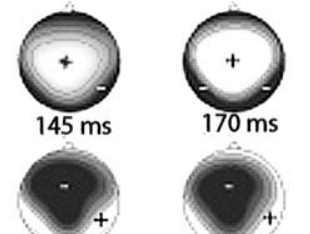

$295 \mathrm{~ms}$
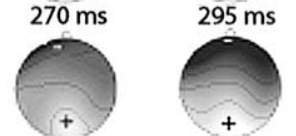

$395 \mathrm{~ms} \quad 420 \mathrm{~ms}$
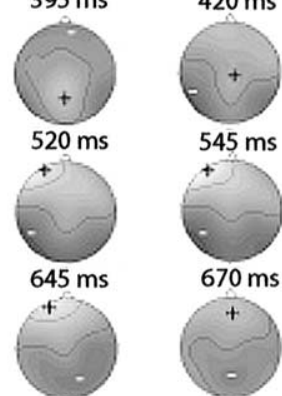

$770 \mathrm{~ms}$

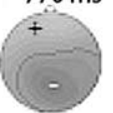

$895 \mathrm{~ms}$
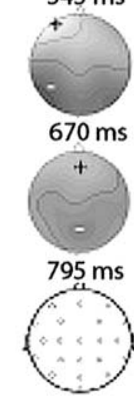

Fig. 1. Potential fields elicited by Chinese words between 70 and $895 \mathrm{~ms}$ after stimulus onset. Maps are shown every $25 \mathrm{~ms}$. Recordings from 29 electrodes as indicated by head scheme. Lines are in steps of $0.6 \mu \mathrm{V}$, dark areas are negative with respect to the average reference. Mean data of 23 subjects. verify that the subjects had actively observed and processed the stimuli. In addition, all subjects filled in semantic differential questionnaires in order to assure reliability of the stimulus material.

The EEG was written continuously to hard-disk, and was visually inspected offline. Eye movement and blink artifacts were detected at the three most frontal electrodes (located at $\mathrm{Fp}_{1}, \mathrm{Fp}_{2}$, and $\mathrm{Fp}_{\mathrm{z}}$ ) that were not included in the analyses, leaving a mean of 141 segments per stimulus class. Artifactfree EEG following stimulus onset was averaged over 1000 $\mathrm{ms}$ according to stimulus class, and was digitally filtered $(1-30 \mathrm{~Hz})$. This resulted in six sets of average evoked potentials for each subject. Evoked components were identified topographically at peak times of the global field power (GFP) curve $[8,13]$, and we will report on components that occurred early after stimulus onset. At individual component latency, the topography of the field distributions was determined for each subject and experimental condition. Component latencies, field strength, and the topographic features of the evoked distributions were compared between stimulus conditions. The significance of the influence of the factors word class $(\mathrm{E}, \mathrm{P}$ or $\mathrm{A})$ and polarity $(+$ or -$)$ was determined by ANOVAs with repeated measurements. Interactions between word class and polarity will be referred to as word dimension.

Varimax-rotated principal components computed on the questionnaire data revealed three components which accounted for about $60 \%$ of the variance $(64.1 \%$ in the questionnaire group; $62.5 \%$ in the EEG group). These components could be clearly identified as Osgood's factors E, P, and A. Factorial similarity exceeded 0.82 for each factor indicating a very high reliability of the dimensions revealed by the semantic differential.

A sequence of potential fields evoked by words between 70 and $895 \mathrm{~ms}$ after the stimulus is illustrated in Fig. 1. For illustration we display the grand means computed over all word classes. There occur several components starting around $95 \mathrm{~ms}$ as reflected by different topographical distribution patterns that occur sequentially. The strength and topography of the evoked fields changes over time. Steps of information processing are reflected by the occurrence of components that are associated with high field strength and stable topography $[8,13]$. This is evident in Fig. 1 around 95, 170, or 270 ms latency. All subjects' individual components were identified at the peak latency of maximal field strength.

We analyzed how latency, field strength, and scalp topography (positive or negative centroid locations in the anterior-posterior or in the left-right direction) of the components were influenced by the semantic dimensions. In the following we will concentrate on effects at small latency that suggest top-down influences on the processing of incoming meaningful information. We note that the potential distributions were similar in all conditions, and their features were modulated by semantic meaning.

The earliest component which was reminiscent of the 
classical P100 appeared between 80 and $130 \mathrm{~ms}$, and its topographical distribution is displayed in Fig. 2. Word polarity had a significant influence on component latency (mean latency for positive words: $108.2 \mathrm{~ms}$; negative words: $105.0 \mathrm{~ms} ; F(1,22)=4.31, P<0.05)$ indicating that the characteristics 'good', 'strong', and 'active' are followed by larger latencies than 'bad', 'weak', and 'passive' (Fig. 2). The strength of the same component (reflected by GFP) showed a tendency to be influenced by word dimension $(F(2,44)=2.81 ; P<0.070)$.

Three significant effects were seen with another component which occurred between 130 and $195 \mathrm{~ms}$ (mean latency $170.4 \mathrm{~ms}$; SD $13.1 \mathrm{~ms}$ ). Its topography is illustrated in Fig. 3A. Field strength was modulated by word dimension $(F(2,44)=5.78 ; \quad P<0.006)$ where 'weak' stimuli yielded largest responses (Fig. 3B).

In addition, the locations of the negative centroids in the anterior-posterior direction displayed a significant interaction between word class and polarity. $(F(2,44)=3.89 ; P<0.03)$. While negative stimuli were systematically located more over posterior areas from $\mathrm{E}$ to $\mathrm{P}$ to $\mathrm{A}$, positive words showed an opposite relation (Fig. 3C).

When topography was compared across hemispheres (i.e. the location of the positive centers of gravity in the leftright direction), a significant influence of word class was observed. P-stimuli elicited scalp activity closest to the midline, and E-stimuli showed a tendency towards the left, while A-stimuli were located over the right hemisphere (Fig. 3D). Although these differences were small, they were consistent over subjects and statistically significant $(F(2,44)=3.56, P<0.037)$.

A

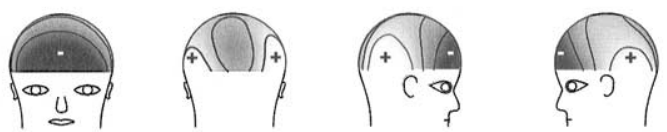

\section{B}

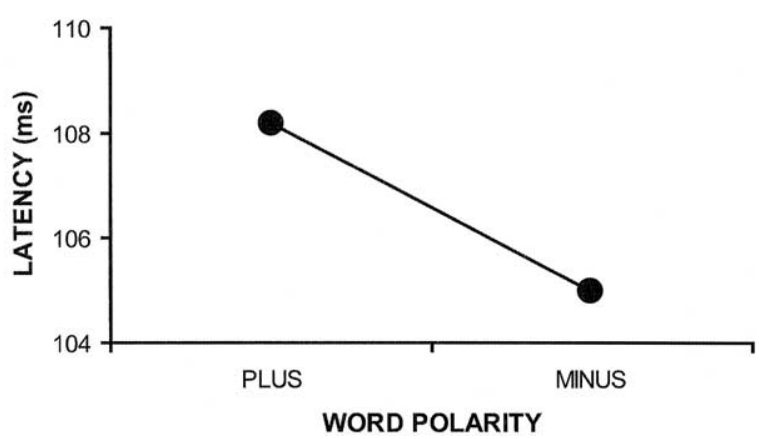

Fig. 2. Effects of semantic meaning on a component occurring between 80 and $130 \mathrm{~ms}$ latency. (A) Scalp topography as seen from front, back, right and left side of the head. Lines are in steps of $0.6 \mu \mathrm{V}$, dark areas are negative with respect to the average reference. (B) Significant effects of word polarity on component latency. Mean data of 23 subjects.
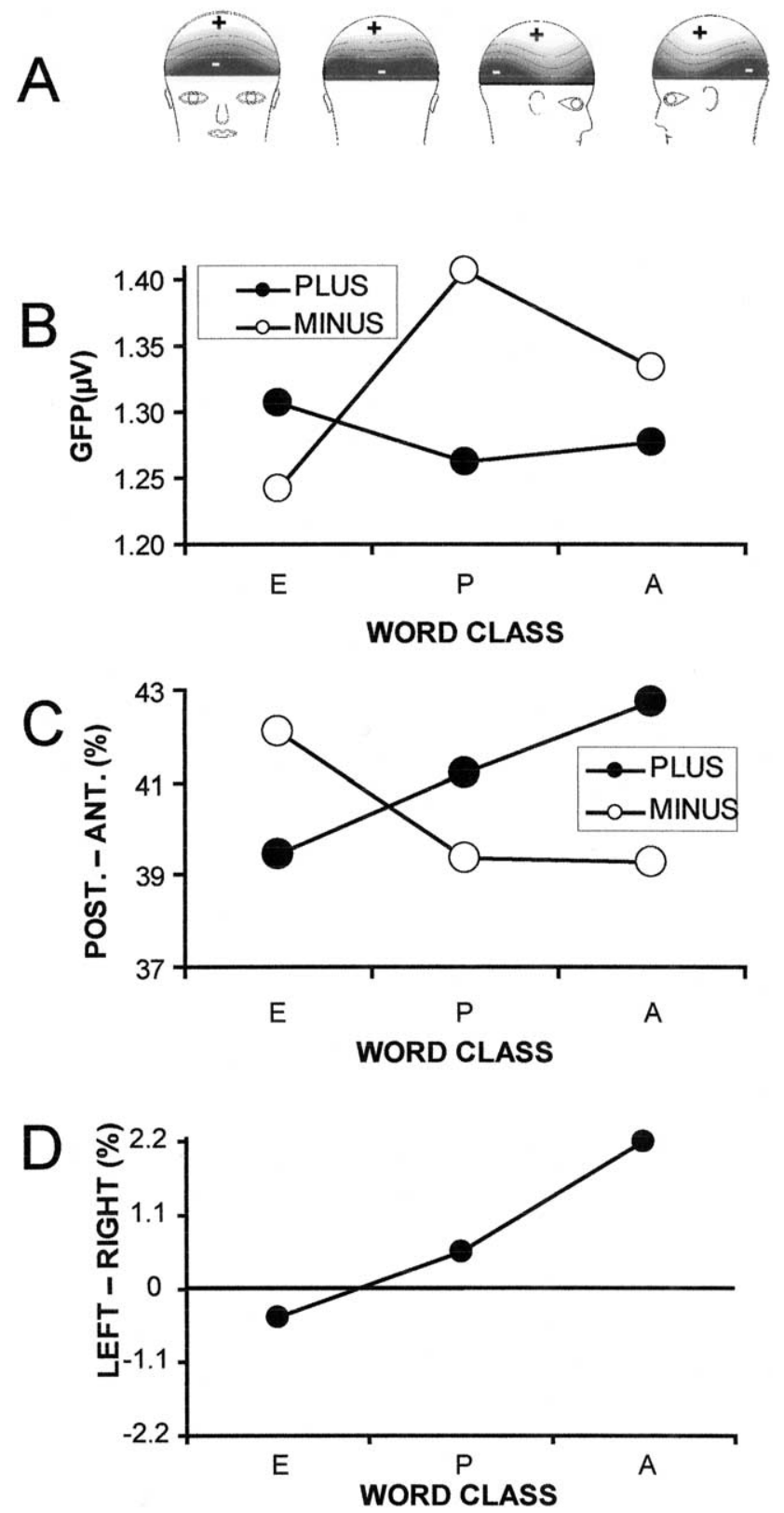

Fig. 3. Effects of semantic meaning on a component occurring between 130 and $195 \mathrm{~ms}$ latency. (A) Scalp topography as seen from front, back, right and left side of the head. Lines are in steps of $0.6 \mu \mathrm{V}$, dark areas are negative with respect to the average reference. (B) Influence of word dimension on GFP. (C) Locations of the negative centroids in the anteriorposterior direction. There is a significant interaction between word class and polarity. The ordinate indicates scalp location in percentages of the nasion-inion distance. (D) Locations of the positive centers of gravity in the left-right direction. There is a significant influence of word class with $\mathrm{E}$ stimuli lateralized over the left and A stimuli over the right hemisphere. The ordinate indicates scalp location in percentages of the nasion-inion distance, 0 corresponds to the midline. Mean data of 23 subjects.

The analysis of the behavioral questionnaire data resulted in the same semantic dimensions as described earlier $[9,10]$, and were very similar to those obtained in German subjects [14]. We could confirm that the dimension E explains most of the variance ( $>30 \%$ in each group). Our electrophysio- 
logical data illustrate that internal representations of semantic meaning of verbal stimulus material can be assessed by analysis of evoked potentials as has been reported with single channel recordings [2] or in topographical recordings [14].

There was a significant effect of semantic meaning on brain electric activity occurring as early as $80 \mathrm{~ms}$ latency. This points to fast processing in higher-level neural networks which may receive the information in parallel and in turn condition the visual cortex. Thus, semantic meaning classified in higher-level structures might represent top-down processing mechanisms. This early component, however, is topographically different from the classical P100 component [14].

Differences were observed with component latency that corresponds to processing time and response strength indicating amount of synchronous activation of cortical neuronal assemblies. In addition to these effects we also describe topographical differences that indicate that not identical generator populations respond to verbal stimuli stemming from different semantic classes. The findings that: (1) brain activity elicited by words is influenced by semantic meaning; and (2) that early processing steps are related to language processing confirms earlier findings on German subjects [14]. Both components presented here are very similar to those reported earlier [14]: the potential distributions display a highly significant correlation of 0.80 at $95 \mathrm{~ms}$ and 0.81 at $170 \mathrm{~ms}$ latency $(P<0.001)$. Of course, the effects are not identical, but the congruence is quite remarkable indicating that the processing of affective meaning of verbal material is universal: Chinese and German language is vastly different, not only according to linguistic categories but there are also large cultural differences. Thus, the E-P-A dimensions of the semantic differential may be regarded as robust and culture-free.

Early semantic effects in the order of about $100 \mathrm{~ms}$ may come as a surprise since most electrophysiological experiments on language processing have reported much larger latencies $[1,7,18]$. We note, however, that subjects are able to read and comprehend words very fast: when words are shown with rapid serial visual presentation, processing time drops well below 100 ms [12] ( discussed in Ref. [15]). In addition, components with latencies in the order of $100 \mathrm{~ms}$ reflect cortical activation as has been demonstrated in studies on 3D perception that indisputably triggers cortical processing [16]. Further evidence stems from intracranial evoked potential recordings in humans [4], and such early brain activity is also sensitive to task relevance [17].

Our results illustrate that significant differences in scalp topography between semantic classes are not restricted to late 'cognitive' components, but brain activity in primary visual areas is affected by semantic meaning of verbal stimuli suggesting 'top-down' mechanisms which confirms the results of a similar study on German subjects.

\section{Acknowledgements}

Supported by DAAD D / 9922885 and NSC. We thank Ms Y.-R. Lin for assistance with data collection and primary analysis, and Ms C. Seidler for help with preparing the figures.

\section{References}

[1] D. Brandeis, D. Lehmann, C.M. Michel, W. Mingrone, Mapping event-related brain potential microstates to sentence endings, Brain Topogr. 8 (1995) 145-159.

[2] R.M. Chapman, J.W. McCrary, J.A. Chapman, J.K. Martin, Behavioral and neural analyses of connotative meaning: word classes and rating scales, Brain Lang. 11 (1980) 319-339.

[3] S. Coulson, M. Kutas, Getting it: human event-related brain response to jokes in good and poor comprehenders, Neurosci. Lett. 316 (2001) $71-74$.

[4] A. Ducati, E. Fava, E.D.F. Motti, Neuronal generators of the visual evoked potentials: intracerebral recording in awake humans, Electroenceph. Clin. Neurophysiol. 71 (1988) 89-99.

[5] K.S. Hamsher, Handedness Questionnaire, University of Wiscosin Medical School, Department of Neurology, Milwaukee, 1981.

[6] C.R. Huang, K.J. Chen, Z.M. Gao, F.Y. Chen, Z.Z. Shen, Chinese Knowledge Information Processing Group Technical Report no. 9801: Word Frequency Dictionary, CKIPG, Academia Sinica, Taipei, 1998.

[7] M. Kutas, S.A. Hillyard, Reading senseless sentences: brain potentials reflect semantic incongruity, Science 207 (1980) 204-206.

[8] D. Lehmann, W. Skrandies, Reference-free identification of components of checkerboard - evoked multichannel potential fields, Electroenceph. Clin. Neurophysiol. 48 (1980) 609-621.

[9] C.E. Osgood, W.H. May, M.S. Miron, Cross-cultural Universals of Affective Meaning, University of Illinois Press, Urbana, IL, 1975, 486 pp..

[10] C.E. Osgood, G.J. Suci, P.H. Tannenbaum, The Measurement of Meaning, University of Illinois Press, Urbana, IL, 1957.

[11] F. Pulvermüller, B. Mohr, H. Schleichert, Semantic or lexicosyntactic factors: what determines word-class specific activity in the human brain?, Neurosci. Lett. 275 (1999) 81-84.

[12] G.S. Rubin, K. Turano, Reading without saccadic eye movements, Vision Res. 32 (1992) 895-902.

[13] W. Skrandies, The upper and lower visual field of man: electrophysiological and functional differences, Prog. Sens. Physiol. 8 (1987) $1-93$.

[14] W. Skrandies, Evoked potential correlates of semantic meaning: a brain mapping study, Cogn. Brain Res. 6 (1998) 173-183.

[15] W. Skrandies, Early effects of semantic meaning on electrical brain activity, Behav. Brain Sci. 22 (1999) 301-302.

[16] W. Skrandies, The processing of stereoscopic information in human visual cortex: Psychophysical and electrophysiological evidence, Clin. EEG 32 (2001) 152-159.

[17] W. Skrandies, R.M. Chapman, J.W. McCrary, J.A. Chapman, Distribution of latent components related to information processing, Ann. N.Y. Acad. Sci. 425 (1984) 271-277.

[18] H. Weyerts, T.F. Münte, H.G.O.M. Smid, H. Heinze, Mental representations of morphologically complex words: an event-related potential study with adult humans, Neurosci. Lett. 206 (1996) $125-128$. 